\title{
Cuantificación del Valor Emocional. El caso de Unión de Cooperativas Agroalimentarias de Navarra (UCAN)
}

\section{Maite Ruiz-Roqueñi}

RESUMEN: El presente trabajo se enmarca en un proyecto de mayor dimensión, que tiene por objetivo monetizar el Valor Social que generan, o destruyen, las diversas organizaciones y que cuenta desarrolladas a día de hoy con tres propuestas complementarias: Un modelo de contabilidad social del valor integral denominado, Modelo Poliédrico; una metodología procesual, basada en el mencionado modelo poliédrico, denominada SPOLY y la existencia de cierta estandarificación de variables de valor social, así como de los proxys que permiten la monetización de los outputs con ellas vinculados (Retolaza et al., 2014, 2015 y 2016).

En línea con dicho modelo de Contabilidad Social, se aborda una nueva dimensión o ecosistema del valor, el valor emocional, y se propone un instrumento para su medición de manera cuantitativa. La propuesta realizada viene determinada por la identificación de un índice, que funcionaría como un factor corrector, permitiendo ajustar al alza o a la baja, el valor social integral. Una adaptación del modelo servqual (Parasuraman, et al., 1988), permitirá la objetivación del factor corrector y la amplitud del rango de corrección, a aplicar al Valor Social Integrado. La aplicación práctica al caso concreto de la Unión de Cooperativas Agroalimentarias de Navarra (UCAN) permitirá cuantificar el valor emocional generado por la entidad, posibilitando su integración con el valor económico y social.

PALABRAS CLAVE: Teoría de stakeholder, contabilidad social, monetización, valor social, valor emocional.

CLAVES ECONLIT: M140, P130, Q130.

Cómo citar este artículo/How to cite this article: RUIZ-ROQUEÑI, M. (2020): “Cuantificación del Valor Emocional. El caso de Unión de Cooperativas Agroalimentarias de Navarra (UCAN)", CIRIEC-España, Revista de Economía Pública, Social y Cooperativa, 100, 155-175. DOI: 10.7203/CIRIEC-E.100.18067.

Correspondencia: Maite Ruiz-Roqueñi, maite.ruiz@ehu.eus, Grupo de Investigación ECRIEthics in Finance and Social Value, Universidad del País Vasco (UPV/EHU), Instituto de Economía Aplicada a la Empresa, Universidad del País Vasco (UPV/EHU), ORCID: 0000-0002-0514-0817. 


\section{Expanded abstract}

\section{Quantification of Emotional Value. The case of Union of Agricultural Cooperatives of Navarra (UCAN)}

\section{Objectives}

The current economic and financial accounting systems provide adequate information, which facilitates the understanding of the economic value generated by an organisation, although they do not allow the social value generated by it to be visualised. Incomplete measurement of value and in particular exclusion of social value could act as a deterrent to implementing people-centred management or social approaches, especially if economic-financial result continues to be interpreted as the most important or even the only valid criterion of success in management (Freeman et al., 2010). This is especially harmful for those organisations linked to the social economy, because of their value system and founding philosophy. As stated in Social Economy Europe (2020) "The social economy is made up of a diversity of enterprises and organisations such as cooperatives, mutuals, associations, foundations and social enterprises among other forms that can be specific to each country. They are united around the values of: primacy of people and the social objective over capital, democratic governance, solidarity and the reinvestment of most profits to carry out sustainable development objectives". So, the complete understanding of the social economy and its value requires new ways of measuring organizational success that are adapted to its reality and that make it possible to highlight and manifest its differential elements by facilitating a greater and better understanding.

The current pandemic we are experiencing has demonstrated the need to rethink our economies and reconfigure the key strategic sectors in them. Traditionally undervalued sectors, such as the agricultural sector, have become essential sectors during the pandemic, providing essential needs and guaranteeing food security, demonstrating their roots and commitment to the local community where they are located. It would seem fair to be able to recognise the contribution to social well-being they had made, and not just now, by agricultural cooperatives and in our case, the Union of Agricultural Cooperatives of Navarra (UCAN), under new performance indicators.

Due to the incomplete measurement of value in the organisations and its special incidence in those belonging to the social economy, the present work is framed in a larger project, which aims at the development of social accounting. This project aims to monetise the Social Value generated, or destroyed, by the various organisations. It currently has three complementary proposals: a social accounting model of integral value called the Polyhedral Model; a processual methodology, based on the aforementioned polyhedral model, called SPOLY and the existence of a certain standardisation of social value variables, as well as proxies that allow the monetisation of the outputs, which are linked to them (Retolaza et al., 2014, 2015 and 2016). 
In addition to this model of Social Accounting, this study deals with a new dimension or ecosystem of value, emotional value, and proposes a tool for measuring it in a quantitative way. The proposal made is determined by the identification of an index, which would function as a corrective factor, allowing upward or downward adjustment of the integral social value. An adaptation of the SERVQUAL model (Parasuraman, et al., 1988) will allow the objectification of the corrective factor and the amplitude of the correction range to be applied. Given that the inclusion of the emotional dimension implies a more complex analysis of the value, the aim is to ascertain the extent to which it is feasible to calculate it in a specific organisation, as well as its complementarity and integration with the other values identified by the Social Accounting model (Retolaza, et al., 2013, 2014, 2015 and 2016).

\section{The methodological design}

The work has focused on a single case of intervention, using the case analysis method (Yin, 2014). The case chosen for analysis was that of the Union of Agricultural Cooperatives of Navarra (UCAN). This entity, with 137 members and a history of more than a century, has as one of its main aims "the promotion and organization of services and actions of general interest for agricultural cooperativism, in order to meet the needs and facilitate the development and efficiency in the management of agricultural cooperatives" (UCAN, 2020). The choice of this entity responds to various reasons. The medium size of the organisation and the basic geographical scope of action, centred on Navarra, facilitate the approach to the organisation and its stakeholders. Secondly, due to the trajectory of this organisation, the cooperative model in the food and agriculture sector in Navarra is a clear point of reference in Spain. Finally, and as a key element of the project's success, there was total management involvement in the project, which was essential for the practical application of the measurement methodology in the organisation. The constant search for excellence serves as an incentive for them to explore new measures of success in their management, in coherence with their foundational goals.

\section{Results}

The emotional value and the measurement instrument proposed has allowed us to visualize a more complete image of the value generated by an entity, specifically, the value generated by UCAN. The data obtained supports the adaptation of the social accounting model to the social entities, by putting in value their social contribution. The image provided by UCAN, of the value it generates, measured through the accounting instruments in use and through economic and financial indicators, hides a large part of the value generated by the entity, a value linked to its own specificity as a social entity.

\section{Conclusions}

The proposal made, by allowing the measurement of the emotional value in monetary units, contributes to the integration of the value, in the same unit of measurement, not only the economic value, but also the social and the emotional one. This allows us to think about how 
measuring the performance of organisations affects both the consideration of their excellence (or failure) in management and their contribution or inhibition to the well-being of their stakeholders and society. It indirectly opens up new ways of satisfying the interests of stakeholders without entering into collusion with other interest groups by overcoming the narrow concept of economic value.

The generalization of social accounting in social entities, we understand, is a key element to improve their management in the search for efficiency and social and economic effectiveness. In turn, it offers information to the different agents in the environment to verify the contribution of each entity to the achievement of more global social objectives, by creating a greater level of well-being for the group of stakeholders inside the value generation system of each entity.

\section{Original Value and Limitations}

This is the analysis of a case that correctly illustrates a significant advance in a relevant problem, the monetisation of the emotional value generated by an organisation to its different stakeholders.

The mains limitation of this study are largely due to the analysis of a single case and the lack of analysis of all stakeholders in the organization.

Keywords: Stakeholder Theory, Stakeholder Accounting, Monetization, Social Value, Emotional Value. 


\section{Introducción}

En los últimos años, el cada vez mayor reconocimiento del papel social de las organizaciones ha conllevado la necesidad de intentar dotar de contenido y de consistencia al concepto de valor social y generar modelos de contabilidad que posibiliten su medición. El reconocimiento de la organización, bajo una perspectiva social, conlleva el cuestionamiento de la medida tradicionalmente aceptada de éxito o rendimiento de la organización, al reconocer la legitimidad de la generación de valor hacia los diferentes grupos de interés. Consecuentemente, si bien una organización puede crear un mayor valor en todo el sistema a través del valor generado hacia los diferentes grupos de stakeholders, dicho valor puede ser difícil de detectar utilizando la contabilidad económico financiera o medidas de rendimiento de los accionistas, porque el valor generado y distribuido hacia los accionistas no proporcionaría una imagen completa del valor que dicha organización genera (Retolaza, et al., 2013, 2014, 2015 y 2016).

Si bien, la academia ha prestado escasa atención al proceso de generación y medición del valor social, entendiendo por tal el valor que reciben y/o captan los stakeholders, primando la investigación del valor bajo el prisma de los accionistas o "propietarios", el estudio del valor bajo la perspectiva stakeholder, resulta importante no solo para la investigación sino para la práctica (Harrison et al., 2010 y Harrison y Wick, 2013). Desde una perspectiva práctica, se gestiona lo que se mide (Kaplan \& Norton, 1992) y si no se realiza una medición completa e integral del valor en las organizaciones difícilmente se podrá gestionar en su conjunto. Así, podría suceder que una entidad gestionara con el fin de aumentar la creación de valor económico financiero para el accionista, sin tener en consideración como podrían repercutir sus actuaciones en el valor generado o destruido hacia otros grupos de interés. Adicionalmente, la gestión de las organizaciones, al amparo de esta nueva perspectiva, focalizando la generación de valor para los diferentes stakeholders, abre nuevas vías de acercamiento al propio concepto de "valor", el cual adopta una visión muy limitada, por su vinculación al valor económico, (Hausman \& McPherson, 2006; Satz, 2010). Muestra de ello es el estudio del valor desde la perspectiva de los consumidores (Sweeney \& Soutar, 2001; Smith \& Colgate, 2007; Zellweger \& Astrahan, 2008), o desde el ámbito de la calidad o los trabajadores (Lewis \& Gabrielsen, 1998; Goetsch \& Davis, 2014).

Así, en la primera parte del trabajo, ofrecemos una reflexión, sobre el propio concepto de valor y argumentamos que el enfoque de valor hacia los stakeholders, no se debería limitarse a ampliar la perspectiva de valor de un grupo de interés hacia los otros, no se pretende trasladar la perspectiva de valor económico del accionista al conjunto de stakeholders, sino ampliar la perspectiva de valor, identificando una nueva dimensión y su medición, el ecosistema del valor emocional. Con ello, entendemos se amplían las posibilidades de aumentar y visibilizar el valor generado por las organizaciones. Por una parte, al ampliar los públicos a los que se puede generar valor. Por otra parte, al posibilitar gestionar para generar valor en una dimensión diferente a la económica.

Dado que uno de los principales objetivos de la dirección estratégica es explicar porque unas organizaciones muestran un mejor desempeño que otras (Rumelt, Schendel \& Teece, 
1994), la identificación del valor socioemocional generado por la organización, entendemos sería un mejor indicador de su desempeño, al ampliar la información proporcionada, dando cabida al valor social recibido por los stakeholders y en todas sus dimensiones, incluida la emocional. De hecho, la medición incompleta del valor social podría actuar como un elemento disuasorio para implementar enfoques de gestión centrados en las personas o en enfoques sociales, especialmente si se sigue interpretando el resultado económico financiero como el criterio de éxito más importante en la gestión o incluso el único válido (Freeman et al., 2010). Esto resulta especialmente lesivo para aquellas organizaciones vinculadas a la economía social, por su sistema de valores y filosofía fundacional. Tal y como recoge la Social Economy Europe (2020) "La economía social está compuesta por una diversidad de empresas y organizaciones tales como: cooperativas, mutuas, asociaciones, fundaciones y empresas sociales entre otras, unidas en torno a los valores de: la primacía de las personas y del objetivo social sobre el capital, la gobernabilidad democrática, la solidaridad y la reinversión de la mayoría de las ganancias para llevar a cabo objetivos de desarrollo sostenible". Resulta claro, que la completa comprensión de la economía social y su puesta en valor exige nuevas formas de medición del éxito organizacional que se adecuen a su realidad y que posibiliten poner de relieve y manifiesto sus elementos diferenciales facilitando una mayor y mejor compresión.

La actual pandemia ha demostrado la necesidad de repensar nuestras economías y de reconfigurar los sectores estratégicos clave en la misma. Sectores tradicionalmente poco valorados, como el sector agropecuario, se han convertido en sectores esenciales durante la misma, al proporcionar bienes de primera necesidad y garantizar la seguridad alimentaria, demostrando su arraigo y compromiso con la comunidad local donde se ubican. Parecería justo poder reconocer la contribución en el bienestar social que han proporcionado, y no solo ahora, las cooperativas agroalimentarias y en nuestro caso, la Unión de Cooperativas Agroalimentarias de Navarra (UCAN), bajo nuevos indicadores de desempeño.

Ante el problema ya expuesto, de la medición incompleta de valor en las organizaciones y su especial incidencia en aquellas pertenecientes a la economía social, el trabajo se centra en la dimensión emocional del valor y su integración en el modelo de contabilidad social, desarrollado por Retolaza, San José y Ruiz-Roqueñi (Retolaza et al., 2013, 2014, 2015 y 2016), definiendo un proceso para su cuantificación. La propuesta realizada viene determinada por la identificación de un índice, que funcionaría como un factor corrector, permitiendo ajustar al alza o a la baja, el valor social integral. Una adaptación del modelo servqual (Parasuraman, et al., 1988) permitirá la objetivación del factor corrector y la amplitud del rango de corrección a aplicar. El objetivo final, es su aplicación al caso concreto de la Unión de Cooperativas Agroalimentarias de Navarra (UCAN), con el fin de poder cuantificar el valor emocional que dicha entidad genera.

El trabajo contribuye a desarrollar la contabilidad social al proporcionar herramientas de medición del valor más completas, incorporando la medición de la dimensión emocional. La propuesta realizada, al permitir medir el valor emocional en unidades monetarias, contribuye a la integración del valor, en una misma unidad de medida, tanto el valor económico, el social como el emocional. Así, posibilita la reflexión sobre cómo repercute la medición del rendi- 
miento de las organizaciones tanto en la consideración de su excelencia (o fallo) en la gestión como en su contribución o inhibición al bienestar de sus stakeholders y de la sociedad. De manera indirecta, abre nuevas vías para posibilitar la satisfacción de los intereses de los stakeholders sin entrar en colusión con otros grupos de interés, al superar la concepción estrecha del valor económico.

El artículo se divide en cinco partes, siendo la primera esta misma introducción. En la segunda se realiza una revisión del marco teórico en relación al valor; y en la tercera, se justifica el método utilizado. En la sección cuarta se exponen los resultados. La última sección recoge las conclusiones, las limitaciones y las futuras líneas de investigación.

\section{El marco conceptual}

La creación de valor, por parte de las organizaciones, es un concepto central y muy importante que genera, no obstante, escasa aquiescencia respecto a qué es el valor, así como cuál es el proceso de creación y de distribución o captación, necesario para determinar su posterior medición, objeto del presente trabajo.

\section{Del valor de los stakeholders al valor para los stakeholders}

Uno de los elementos, que guía la diversidad de interpretaciones del valor, es la diversidad de perspectivas desde las que el valor puede ser estudiado, atendiendo a las personas u organizaciones para las cuales el valor va a ser creado y/o las que son fuentes generadoras del mismo. Tal y como afirma Woodall (2003), referido a los consumidores, el termino valor del consumidor tiene muchos significados, pero dos dominan: valor para el consumidor (valor percibido o valor recibido por el consumidor) y valor para la empresa (valor del consumidor o customer lifetime value).

En el área de la gestión empresarial, el estudio de la generación de valor se ha focalizado generalmente en el valor económico que la empresa genera, siendo el destinatario de este valor los propietarios de la misma (Friedman, 1972). Atendiendo esta perspectiva, se ha ido desarrollando un sistema de contabilidad, con el fin de transmitir una imagen fiel del valor generado por la empresa para el accionista, constituyendo un reflejo del buen (mal) hacer en gestión por la organización. Esta perspectiva ha guiado sobremanera la investigación sobre el valor generado por la empresa para otros grupos de interés. Si bien, existen numerosos estudios que ponen su foco en la generación de valor vinculado a los stakeholders, muchos de ellos se focalizan en la generación de valor hacia los grupos de interés, pero como un medio para generar, en última instancia, valor para los accionistas, (Laplume, et al., 2008). La literatura empírica revisada al respecto (Freeman et al., 2010), trata de consolidar la relación positiva entre la gestión social vinculada a los grupos de interés y el desempeño financiero, el cual 
suele ser medido en términos de retorno financiero (Orlitzky et al., 2003). En consecuencia, nos encontramos que parte de la literatura empírica, que justifica una gestión orientada hacia la creación de valor a los stakeholders, refuerza per se, la idea de que el retorno financiero es la medida más importante en la generación de valor creado por la empresa. La premisa subyacente, en estos estudios, mantiene que las medidas económico financieras al uso capturan el valor creado, a través de la gestión de los intereses de los stakeholders, eludiendo la necesidad de visibilizar, que gran parte del valor generado no para la empresa, sino para los stakeholders, no puede ser capturado a través de estas medidas económicas exclusivamente (Harrison \& Wicks, 2013).

Estudios afines al área de recursos humanos y de marketing, focalizados en el estudio de la creación de valor, vinculados a los trabajadores y los clientes respectivamente, adoptan esta misma perspectiva y avalan esta relación. La gestión por parte de la empresa hacia estos grupos de interés, con el fin de generarles valor y construir relaciones con ellos, es guiada con el fin de capturar el valor que generan para la empresa. Así, el análisis de valor vinculado a dichos grupos se realiza desde la perspectiva de la empresa (propietario/accionista) y viene determinado por conceptos tales como el valor capital de los clientes y/o el capital intelectual (Beckers, Van Doorn \& Verhoef, 2018; Venkatesan \& Kumar, 2004; Sullivan, 2000 y Zeghal \& Maaloul, 2010).

El elemento común de estos trabajos es el foco que guía el análisis para entender la creación de valor, la primacía del accionista. Conviene, no obstante, recordar que su justificación viene dada por la economía ortodoxa, que considera que bajo una serie de supuestos (competencia perfecta, nula existencia de barreras de entrada y salida, nula existencia de bienes libres y/o públicos o no externalidades) se llegaría a conseguir el óptimo económico, en términos de maximización del valor (económico) social para la economía en su conjunto (Williamson, 1984). Doscientos años de trabajo en economía y finanzas indican que el bienestar social se maximiza cuando todas las empresas en una economía intentan maximizar su propio valor total. Si bien, este objetivo no es incompatible con la gestión a favor de los intereses de los stakeholders, sino necesario para su consecución en el largo plazo (Jensen, 2001).

Desde la perspectiva de la economía social, que es el caso que nos ocupa, parece que existe una mayor delimitación conceptual. En un intento de clarificación del sector, Gui (1991) identifica varios tipos de entidades de economía social: las de interés general y mutualístico. Las de interés general se caracterizan porque los beneficiarios de la actividad económica son diferentes del grupo de personas que controla la organización. Nos encontramos, por tanto, ante un reconocimiento implícito de legitimidad de gestión multistakeholder, ampliando la perspectiva de creación de valor y desvinculando los procesos de creación de valor de la organización con los de creación de valor económico financiero para los aportantes de capital. Por su parte, en las organizaciones de interés mutualístico, los beneficios de la actividad económica recaen en quienes poseen el poder de decisión. Los socios/as de estas entidades pueden ser tan variados como su propósito empresarial, así consumidores, pequeños productores o trabajadores pueden decidir organizarse orientando la gestión de la generación de valor de la entidad a los intereses de los mismos. Este autor instituye como elemento clave diferenciador 
de las organizaciones de economía social el hecho de que las personas beneficiarias de la actividad de la organización y las que poseen el último poder de decisión no son los inversores capitalistas, como sucede en las empresas mercantiles, cuyo concepto de creación de valor se asimila tradicionalmente con la consecución de la máxima rentabilidad posible.

Por su parte, Chaves y Monzón (2018) identifican dos grandes subsectores en la economía social: el subsector de mercado y el de no mercado. El primero de ellos está integrado fundamentalmente por cooperativas, mutualidades u otras entidades que obtienen sus recursos de la venta de bienes y servicios en el mercado. Estas organizaciones, al igual que las mercantiles, desarrollan procesos productivos eficientes, que conduzcan al máximo resultado utilizando los mínimos medios, pero al amparo de un propósito empresarial diferente que condiciona, no solo el ejercicio de la actividad económica en sí misma y la forma en la que se crea el valor, sino la eventual distribución de beneficios o excedentes bajo una perspectiva multistakeholder. El segundo, el subsector de no mercado, lo integrarían las instituciones sin fines de lucro al servicio de los hogares.

A pesar de esta especificidad en la economía social y del propósito de sus organizaciones de crear valor para el conjunto de la sociedad y de sus miembros, las herramientas utilizadas para la medición del valor generado siguen siendo las económico financieras, con foco único en el accionista. Esta perspectiva de estudio, no ayuda a aportar luz, al objetivo buscado de comprender para medir, el valor generado y capturado por y para los diferentes grupos de interés. En el ámbito de la economía social, en entidades con un propósito explicito social, resulta especialmente importante avanzar en una nueva perspectiva de estudio del valor que permita visualizar el valor más allá del generado por las transacciones de mercado y multistakeholder (Lazkano et al., 2019). La creación de valor bajo esta nueva perspectiva y su medición, exige reflexionar, al cambiar la perspectiva sobre quien valora, sobre conceptos tales como, qué es valor y qué es valorable.

\section{El valor}

Para llegar a la cuantificación del valor emocional, resulta necesario clarificar el propio concepto de valor.

Los fundamentos conceptuales del valor de uso y del valor de cambio los podemos encontrar en el campo de le ética, vinculada a los primeros filósofos. Si bien, se suele atribuir a Aristóteles las primeras reflexiones a este respecto, son numerosos los autores y escuelas de pensamiento que lo han ido dotando de contenido a lo largo de la historia del pensamiento económico. Bowman y Ambrosini (2000) definen y diferencian dichos tipos de valor. Así, el valor de uso se refiere a las cualidades específicas del producto/servicio que son percibidas por el consumidor en relación a sus necesidades. El valor de uso es por tanto, un valor percibido por el consumidor, es subjetivo, y es específico de cada persona. El valor de cambio expresa el precio que el consumidor está dispuesto a pagar por él. Viene medido en unidades monetarias y se materializa en el momento del intercambio. Si bien, la definición de ambos conceptos se 
reduce al consumidor, estas concepciones del valor serían extrapolables a cualquier grupo de stakeholders en sus transacciones en el mercado.

La clarificación e identificación de estos conceptos ponen de manifiesto la diferente naturaleza del valor. Así, "la naturaleza del valor de cambio podría ser considerada como una utilidad, basada en el valor embebido en un recurso y como resultado de un proceso de manofactura, que existe como una entidad singular en un momento dado y puede intercambiarse por otras utilidades (o por lo que el cliente está dispuesto a pagar). La naturaleza del valor en uso, en su lugar, es la medida en que un cliente se siente mejor (valor positivo) o peor (valor negativo) a través de las experiencias relacionadas con el consumo" (Grönroos \& Voima, 2013). En este sentido es remarcable el cambio en el concepto de valor desde la perspectiva del consumidor, inicialmente centrado en una perspectiva de estudio orientada a la producción de bienes y servicios donde el valor estaba embebido en el propio producto a la actualidad donde la discusión sobre el valor ha evolucionado desde estas lógicas vinculadas a los bienes físicos a una perspectiva más plural, vinculada a la lógica de los servicios donde adquiere especial relevancia el valor en uso (Vargo, 2008 y Vargo \& Lusch, 2008a, b).

Esta tipología de valor ayuda a entender, tal y como ya hemos mencionado, que la perspectiva desde la que se estudia el valor, afecta a su medición y a su creación. Desde la perspectiva del consumidor, el valor del consumidor es lo que obtiene en relación a lo que tiene que renunciar (costes, sacrificio) (Zeithaml, 1988). Diferentes autores indican que salvo en circunstancias excepcionales, de eficiencia total de los mercados, se generaría un excedente para el consumidor, dado que el precio pagado sería menor que el valor monetario total que estaría dispuesto a pagar por el valor que percibido que recibe (Basch et al.,1987 y Whitehead, 1996). Por su parte, el excedente del productor (accionista o propietario) vendría definido por la diferencia entre el precio cobrado por el vendedor y el coste de los recursos empleados. El excedente que recibirían el resto de stakeholders tanto su cuantía como su reparto vendría dado por el mercado laboral en el caso del trabajador, por el mercado financiero en el caso del proveedor de recursos financieros y por el resto de mercados de recursos (Argandoña, 2011).

Si bien, el valor de cambio es conocido al venir determinado por el mercado, a efectos de expresar cuantitativamente el valor recibido por cada uno de los grupos de interés, en su relación con la empresa, sería preciso poder cuantificar el valor percibido en uso derivado de sus transacciones y o relación con la misma. En relación al consumidor, Collis (1994), argumenta que el valor percibido de uso se podría expresar en términos monetarios, de manera que podría ser considerado como el precio que un consumidor estaría dispuesto a pagar si solo hubiera un oferente. Es decir, para poder cuantificar el valor percibido de uso se tendrían que dar dos condiciones, la primera, que se podría realizar una evaluación del valor del producto, la segunda, que debería venir acompañada de una disposición a realizar el pago. Si bien, la extrapolación de dicho cálculo en relación al valor experimentado por otros stakeholders no está muy desarrollada, en el ámbito del análisis coste beneficio, existen propuestas metodológicas que abogan por la inclusión del valor moral al cálculo coste beneficio, siempre que haya una disposición al pago, (Zerbe, 2004 y 2006). 
La consideración del valor emocional como un output con posibilidad de ser valorado desde una perspectiva económica en igualdad con otros, se produce desde el momento en que las personas reconocen estar dispuestas a pagar por ellos o a tenerlos en consideración en los intercambios realizados con la organización o en su engagement con la organización. Entendemos que esta perspectiva abre una fructifica línea de investigación tendente a cuantificar el valor emocional, desde la perspectiva subjetiva de cada persona. Su medición, implicaría el diseño de instrumentos ad hoc que contemplen la definición conceptual de lo que se quiere medir, la identificación de las dimensiones subyacentes al concepto y el desarrollo de ítems fiables y válidos para medir cada una de las dimensiones.

En coherencia con el modelo de contabilidad social en el que se integra, la cuantificación del valor social no será obtenido exclusivamente a través de la valoración subjetiva del individuo. Dado el carácter práctico con el que nace esta investigación, la elección del proceso de cuantificación del valor emocional, que presentamos en el siguiente apartado, ha venido limitada por los costes y recursos necesarios para su medición. Así, nos hemos servido de herramientas ya desarrolladas y testadas en el ámbito de la calidad del servicio, que, al igual que el concepto que se pretende medir, resultan de naturaleza abstracta, si bien, son herramientas conocidas y utilizadas en el ámbito de gestión.

\section{El método}

Tal y como ya se ha puesto de manifiesto, el objetivo final del presente trabajo es calcular el valor emocional generado por UCAN, expresando dicho valor en unidades monetarias. Dado que la inclusión de la dimensión emocional implica realizar un análisis más complejo del valor, se pretende constatar hasta qué punto es factible su cálculo en una organización concreta, así como su complementariedad e integración con el resto de valores identificados por el modelo de Contabilidad Social (Retolaza, et al., 2013, 2014, 2015 y 2016). El aprendizaje obtenido desde esta propuesta y su aplicación en la práctica permitirán seguir avanzando hacia medidas más completas y complejas del valor.

El trabajo se ha centrado en un caso único de intervención, utilizando el método de análisis del caso (Yin, 2014). El método se ha elegido por su eficacia demostrada en el desarrollo de nuevos conocimientos teóricos y prácticos comprobables y con validación empírica (Eisenhardt \& Graebner, 2007).

El caso elegido para el análisis ha sido el de la Unión de Cooperativas Agroalimentarias de Navarra (UCAN). Esta entidad, con 126 socios y una trayectoria de más de un siglo, tiene como uno de sus fines principales "la promoción y organización de servicios y actuaciones de interés general para el cooperativismo agrario, encaminados a atender las necesidades y facilitar el desenvolvimiento y la eficacia en la gestión de las cooperativas agroalimentarias" (UCAN, 2020). La elección de dicha entidad responde a diversas razones. La dimensión de tamaño medio de la organización y el ámbito geográfico básico de actuación, centrado en Navarra, facilitan el acercamiento a la organización y sus grupos de interés. En segundo lugar, por la trayectoria de esta 
organización, el modelo cooperativo agroalimentario navarro es un claro referente en el ámbito estatal. Por último, y como un elemento clave de éxito del proyecto, existía una implicación total de la dirección en el mismo, hecho imprescindible para la aplicación práctica de la metodología de medición en la organización. La búsqueda constante en la excelencia les sirve de revulsivo para explorar nuevas medidas de éxito de su gestión, en coherencia con sus fines fundacionales.

La metodología elegida para calcular la medición del valor emocional, se integra en el modelo de contabilidad social, que se basa en cuatro supuestos: El action research, la teoría de los stakeholders, la perspectiva fenomenológica y el valor razonable basado en la lógica borrosa. Para un mayor detalle, véase los trabajos que desarrollan este sistema de contabilidad social (Retolaza et al., 2013, 2014, 2015 y 2016).

El modelo de contabilidad social nació con el propósito de formar un soporte conceptual lo suficientemente sólido que permitiera avanzar hacia medidas más completas del valor. Así, permite visibilizar las diferentes categorías o ecosistemas de valor, identificadas a día de hoy, y que explicamos a continuación.

\section{Valor Social de mercado}

Es una primera aproximación a la medición del valor social y su distribución. Su cálculo se asimila a la propuesta realizada por la Gonzalo \& Pérez (2017), utilizando el análisis del valor añadido y por el GRI4 (GRI, 2013) sobre el valor creado y distribuido. Refleja el valor social originado en las transacciones de mercado entre la empresa, sus clientes, la Administración Pública y otros proveedores de recursos. Si bien, es cierto que su cálculo supone una mejora en la información proporcionada por las organizaciones sobre el valor distribuido a diversos stakeholders, el valor identificado queda reducido al valor meramente económico y al que se materializa a través de transacciones que se producen en el mercado. Pese a presentar una visión estrecha tanto del grupo de stakeholders como del valor visibilizado, presenta la virtud de la facilidad de la obtención de la información necesaria para su cálculo, ya que parte de datos ya identificados y recogidos por las organizaciones y se puede obtener de sus registros contables.

Este análisis asume en general, los principios de creación de valor de la perspectiva neoclásica. La creación de valor es independiente de su distribución. La creación de valor se focaliza en generar excedente para el propietario (accionista). La visibilización del valor de otros grupos de interés, se obtiene al trasladar la perspectiva de valor económico del accionista al conjunto de stakeholders, obviando una interpretación más amplia del valor, posible desde la perspectiva de los stakeholders.

\section{Valor Social de no mercado}

Supone otra categoría o ecosistema de valor y por tanto ayuda a visibilizar con mayor precisión el valor social. Identifica el valor social originado en las transacciones, no realizadas a través de mercado, entre la empresa y sus stakeholders. Es de sumo interés para aquellas 
entidades en las que el valor generado y distribuido se hace mayoritariamente a través de mecanismos de no mercado (Sandel, 2012).

Su medición se realiza desde la perspectiva stakeholder, entendiendo por tal aquellos grupos de personas u organizaciones a los que la organización genera o puede generar valor. Desde una perspectiva fenomenológica, serán ellos/as quienes identifiquen las fuentes o variables de valor, requiriendo del diálogo con los diferentes stakeholders para obtener dicha información. Dado que en este caso es frecuente que no exista un valor de mercado, dichas variables de valor expresadas en forma generalista deberán ser reformuladas en términos de indicadores que se correspondan con outputs mesurables por la organización, donde una vez seleccionados los proxys, se valorará los outputs en unidades monetarias.

\section{Valor emocional}

Si bien el desarrollo inicial del modelo de contabilidad social identificaba esta categoría de valor y remarcaba su importancia, no se procedía a su valoración y cuantificación al exigir un procedimiento de medición diferente, no solo por la subjetividad del concepto, sino por la intangibilidad del mismo. Esto hizo que, en el momento inicial, se abordara desde una perspectiva descriptiva, recogiendo las situaciones y las emociones que evocaban los diferentes grupos de stakeholders. No obstante, el carácter cualitativo, de la información recogida, dificultaba el objetivo pretendido de integración con el resto de categorías identificadas. Y lo que era más importante, había una parte del valor que no afloraba ni quedaba recogido en el modelo de contabilidad social.

Con el fin de poder avanzar en la valoración y cuantificación del valor emocional, y en su integración con el resto de categorías del modelo, se presentó en un curso de verano de la UPV/ EHU la propuesta aquí recogida (Retolaza et al. y Ruiz-Roqueñi, 2018) que viene dada por la identificación de un índice que funcionaría como un factor corrector, permitiendo ajustar al alza o a la baja, el valor social de mercado y de no mercado ya calculado (Guasch 2015). La perspectiva que subyace a esta propuesta, es que los valores monetarios (valor de cambio) previamente identificados en las transacciones de mercado y no mercado, no recogen de manera fiel todo el valor generado a los diferentes stakeholders (valor en uso). Por ejemplo, el valor que dos empleados reciben en su relación con una organización podría ser diferente en función del valor que cada trabajador percibiera en términos de sentimientos y emociones de la entidad, a pesar de que el valor social de mercado, cuantificado por el salario neto, para ambos fuera idéntico. Así, aunque el valor social de mercado que capturan ambos trabajadores con un mismo salario sería el mismo, el valor recibido debería ser ajustado con un factor corrector al alza (baja) en el caso de que las emociones y sentimientos fueran positivos (negativos), para ajustar el verdadero valor recibido por cada uno de ellos. En última instancia, este factor corrector, pondría de manifiesto la diferencia existente entre valor percibido y el precio (valor razonable), dado que, si el precio sería un indicador óptimo del valor, el factor corrector sería cero. 
El factor corrector podría ser único, aplicándose al total del valor social agregado de mercado o de no mercado en su conjunto, o de manera más precisa, identificar un factor corrector para cada uno de los diferentes grupos de interés, en cuyo caso se ajustaría el valor para cada grupo.

Para el cálculo del índice, que actuará como factor corrector, se ha tenido en cuenta la coherencia de la propuesta realizada con los supuestos subyacentes al modelo general de contabilidad social en el que se enmarca la misma, en particular, en lo referente a la propia naturaleza del valor entendido desde una perspectiva stakeholder y la perspectiva fenomenológica desde la cual se determina el valor. Al amparo de estas premisas, se han extrapolado los avances que, desde el área de marketing, se han formulado en relación al concepto valor (Vargo y Lusch, 2008; Vargo et al. 2008) a nuestra proposición. La propuesta concreta se basa en la utilización de la escala de medida del Modelo SERVQUAL (Parasuraman, et al., 1988) adaptada a cada organización, para la obtención de los índices.

Este instrumento de medición cuantitativo de la calidad del servicio, tiene la bondad de identificar diferentes dimensiones subyacentes al concepto, generalizables a cualquier servicio, y proporciona una batería de ítems para medirlas. Su esencia puede resumirse, en que la percepción global que experimenta un cliente (o por extrapolación cualquier grupo de interés) en la relación que establece con una organización es la suma de varios elementos, entre los que se incluyen sensaciones, impresiones, satisfacciones, como parte del proceso que se da en la transacción realizada.

Esta herramienta, ha sido sometida a diversas contrastaciones para asegurar su fiabilidad y su validez hasta llegar a una versión depurada del primer instrumento (Parasuraman et al., 1991). En las sucesivas mejoras, se identificaron 5 dimensiones, que fueron las que utilizamos y aplicamos al caso objeto de estudio:

1) Tangible (tangible).

2) Fiabilidad (reliability).

3) Interés (responsiveness).

4) Seguridad (assurance).

5) Empatía (empathy).

Junto con la identificación del factor corrector por cada grupo de interés, es necesario establecer un rango de amplitud (límite superior o inferior) que permita especificar en qué medida va a corregir dicho factor. Para su cálculo, se debería añadir una cuestión adicional, al cuestionario adaptado con la escala servqual, con el fin de identificar el peso del valor emocional en relación al resto de valores para cada persona.

Así, el acervo de información necesario para la cuantificación del valor emocional, pasa por el cálculo previo del valor social de mercado y de no mercado de dicha entidad. Los datos necesarios para calcular el valor emocional al igual que el valor de no mercado, son datos que emanan directamente de la población objeto de estudio como receptora del valor, es decir los stakeholders de la organización, dado que generalmente no se desprenden de los datos recogidos por la empresa. Con la particularidad de que, en este caso, la información obtenida debe ser representativa de la población objeto de estudio y por tanto se debería utilizar un muestreo representativo. 


\section{El valor socioemocional}

La agregación del valor obtenido en las diferentes categorías o ecosistemas de valor recibe la denominación del valor socioemocional, que incorpora tres tipos de valor de diferente naturaleza medidos una misma unidad de medida.

\section{Resultados}

Siendo el objetivo la monetización del valor emocional de UCAN, en primer lugar, se calculó el valor social generado por la actividad mercantil y no mercantil de la entidad, para calcular el valor social integral anual de la entidad. Una vez obtenido este valor, se aplicó el factor corrector al valor social integral para obtener el valor emocional.

Para el cálculo del Valor Social generado por la actividad mercantil de UCAN, se partió de los datos de la actividad económica, que en su caso se redujeron a: salarios, pagos a la seguridad social, impuestos, retenciones y el resultado del ejercicio. En base a estos datos, se obtuvo que UCAN, a través de su actividad mercantil directa generó, en 2016, un valor para la sociedad (stakeholders) de $291.379 €$, y un retorno a la administración de $107.905 €$. Este mismo análisis se realizó con los proveedores de la entidad, imputando como valor social de mercado indirecto a UCAN, el importe de valor relativo a su facturación. Así, el valor social generado de forma indirecta por la compra a proveedores asciende a $89.039 €$ y el retorno generado para la administración es de $38.882 €$.

Para el cálculo del valor social de no mercado, el modelo de contabilidad social determina un proceso que cuenta con seis fases diferenciadas: 1) configuración del equipo y aprobación del cronograma; 2) Identificación de los grupos de interés a los que la organización presupone que les genera valor; 3 ) Identificación de las variables de valor, entendiendo por tales, los aspectos en los que la organización genera valor a terceros; 4) cuantificación intersubjetiva de los outputs vinculados a cada variable de valor a través de proxys; 5)Integración de resultados, dando lugar al valor denominado valor social integral; 6) Proceso de feedback y mejora continua.

La integración del valor social generado a través de la actividad de mercado (380.418€) con el valor social de no mercado (1.937.727 €), conforma el valor social integral generado por la entidad, que en el caso de UCAN, ascendió en el año 2016 a la cantidad de 2.318.146€.

El Valor Emocional se estimó en base a un cuestionario, basado en el Modelo SERVQUAL, desarrollado por Zeithaml y Berry (1988), y utilizado en el marco de la metodología EFQM. En el estudio se preguntó: 1) sobre la importancia que la entidad tenía para los diferentes stakeholders, para determinar el rango de variabilidad del Valor Social Emocional en relación al Valor Social Integrado; 2) por las dimensiones identificadas en la escala del modelo SERVQUAL con el fin de determinar el factor corrector a imputar sobre el valor social integrado ya calculado. Los resultados obtenidos para las 5 variables valoradas se presentan en la tabla 1. 


\section{Tabla 1. Valor emocional}

\begin{tabular}{cccccr} 
Importancia & $\mathbf{0 , 0 0}$ & $\mathbf{1 , 0 0}$ & $\mathbf{4 , 0 0}$ & $\mathbf{1 1 , 0 0}$ & $\mathbf{4 , 0 0}$ \\
\hline Media & 0,78 & & & & \\
\hline Fiabilidad & 0,00 & 0,00 & 3,00 & 13,00 & $\mathbf{4 , 0 0}$ \\
\hline Respuesta & 0,00 & 1,00 & 7,00 & 7,00 & 5,00 \\
\hline Seguridad & 0,00 & 0,00 & 6,00 & 11,00 & 3,00 \\
\hline Empatía & 0,00 & 0,00 & 5,00 & 9,00 & 6,00 \\
\hline & 0,00 & 1,00 & 21,00 & 40,00 & 18,00 \\
\hline Media & 3,938 & & & & \\
\hline Media & 3,938 & & & & \\
\hline
\end{tabular}

Fuente: elaboración propia.

Los resultados del valor socio emocional, desagregado por categorías o ecosistemas de valor y su distribución entre los diferentes grupos de interés de UCAN para el año 2016 se pueden ver en la siguiente tabla.

\section{Tabla 2. Valor socio emocional}

\begin{tabular}{lccccccc} 
& Sociedad & $\begin{array}{r}\text { Adminis- } \\
\text { tración } \\
\text { pública }\end{array}$ & $\begin{array}{r}\text { Provee- } \\
\text { dor }\end{array}$ & $\begin{array}{r}\text { Trabaja- } \\
\text { dor/a }\end{array}$ & Inversores & $\begin{array}{c}\text { Entidades } \\
\text { sociales }\end{array}$ & Socios \\
\hline $\begin{array}{l}\text { Valor social } \\
\text { de mercado }\end{array}$ & $380.418 €$ & $146.787 €$ & $71.960 €$ & $195.219 €$ & $11.285 €$ & $2.136 €$ \\
\hline $\begin{array}{l}\text { Valor social } \\
\text { de no } \\
\text { mercado }\end{array}$ & $1.937 .727 €$ & $98.710 €$ & $0 €$ & $1.668 €$ & & $93.850 €$ & $1.829 .059 €$ \\
\hline $\begin{array}{l}\text { Valor social } \\
\text { integrado }\end{array}$ & $2.318 .146 €$ & $245.497 €$ & $71.960 €$ & $196.887 €$ & $11.285 €$ & $93.850 €$ & $1.831 .195 €$ \\
\hline $\begin{array}{l}\text { Valor } \\
\text { emocional }\end{array}$ & $708.481 €$ & & & & & & \\
\hline $\begin{array}{l}\text { Valor socio- } \\
\text { emocional }\end{array}$ & $3.026 .627 €$ & & & & & & \\
\hline
\end{tabular}

Fuente: elaboración propia. 


\section{Conclusiones}

La conceptualización de la creación de valor desde la perspectiva neoclásica, nos ayuda a entender la visión tan estrecha del valor que tenemos en la actualidad. Si bien, su simplificación ha facilitado la construcción de modelos, útiles a nivel de investigación, entendemos ha conllevado a enmascarar elementos críticos en la medición del valor, al ocultar dimensiones de valor que superen la estrictamente económica y los habituales indicadores de rentabilidad. Si bien es innegable, que el valor económico es una dimensión fundamental y común a la generación de valor, con validez para el conjunto de stakeholders, la mayoría de las personas reciben otros elementos que también se constituyen como generadores de valor para ellas.

Dada la cada vez mayor aceptación de la función social de las organizaciones y aceptando su capacidad para generar valor en dimensiones diferentes a la económica, una valoración adecuada del valor requiere que se incluya el valor emocional como una dimensión o categoría del valor. La medición del valor emocional en UCAN, nos ha permitido constatar que es posible su medición.

El valor emocional y el instrumento de medición propuesto nos ha permitido visualizar una imagen más completa del valor que genera una entidad, en concreto, el valor generado por UCAN. Los datos obtenidos refrendan la adecuación del modelo de contabilidad social a las entidades sociales, al poner en valor su contribución social. La imagen proporcionada por UCAN, del valor que genera, medido a través de los instrumentos de contabilidad al uso y a través de indicadores económicos financieros, ocultan una gran parte del valor generado por la entidad, valor vinculado a su propia especificidad como entidad social.

La generalización de la contabilidad social en entidades sociales, entendemos es un elemento clave para mejorar su gestión en aras a la búsqueda de la eficiencia y eficacia social y económica. Por su parte, ofrece información a los distintos agentes del entorno para verificar la contribución de cada entidad a la consecución de objetivos sociales más globales, al crear un mayor nivel de bienestar para el conjunto de stakeholders dentro del sistema de generación de valor de cada entidad.

La aplicación de la propuesta de medición a un caso único, valida la utilidad el modelo y su aplicabilidad, si bien, imposibilita la realización de análisis comparativos. Las limitaciones del trabajo se deben en gran medida al análisis de un único caso y a la falta de análisis de la totalidad de grupos de interés de la organización. Dado el carácter práctico con el que nace esta investigación, la elección de los instrumentos de cuantificación del valor vienen, en parte, limitados por los costes y recursos necesarios para su medición, sin perjuicio de la rigurosidad de su análisis. Así, una futura línea de investigación deberá conllevar una mejora y simplificación del proceso, con el fin de facilitar su utilización por parte de un mayor número de entidades. En esta línea, se valora la exploración de la reputación como factor corrector. Análisis específicos sobre la relación entre el rendimiento económico financiero y el rendimiento social, apuntan a la reputación como un mediador importante en la relación positiva entre las medidas de desempeño social y las financieras (Orlitzky et al., 2003). 


\section{Bibliografía}

ARGANDOÑA, A. (2011): "Stakeholder theory and value creation", Working Paper IESE, 9, IESE, Business School, Barcelona, Spain.

BACH, G.L., FLANAGAN, R., HOWELL, J., LEVY, F. \& LIMA, A. (1987): Microeconomics. 11ed., Prentice-Hall, Englewood Cliffs, NJ.

BECKERS, S.F., VAN DOORN, J. \& VERHOEF, P. C. (2018): “Good, better, engaged? The effect of company-initiated customer engagement behavior on shareholder value", Journal of the Academy of Marketing Science, 46(3), 366-383, DOI 10.1007/s11747-017-0539-4.

BOWMAN, C. \& AMBROSINI, V. (2000): "Value creation versus value capture: towards a coherent definition of value in strategy", British Journal of Management, 11(1), 1-15, D0I: 10.1111/14678551.00147.

CHAVES, R. \& MONZÓN, J.L. (2018): “La economía social ante los paradigmas económicos emergentes: innovación social, economía colaborativa, economía circular, responsabilidad social empresarial, economía del bien común, empresa social y economía solidaria", CIRIEC-España, Revista de Economía Pública, Social y Cooperativa, 93, 5-50, D0I:10.7203/CIRIEC-E.93.12901

COLLIS, D. (1994): "How valuable are organizational capabilities?", Strategic Management Journal, 15, 143-152, DOI: 10.1002/smj.4250150910.

EISENHARDT, K.M. \& GRAEBNER, M.E. (2007): "Theory building from cases: opportunities and challenges", Academy of Management Journal, 50(1), 25-32, DOI: 10.5465/amj.2007.24160888.

FREEMAN, R.E., HARRISON, J.S., WICKS, A.C., PARMAR, B.L. \& DE COLLE, S. (2010): Stakeholder theory: The state of the art, Cambridge University Press, Cambridge.

FRIEDMAN, M. (1972): "The social responsibility of business", The New York Times Magazine.

GLOBAL REPORTING INITIATIVE (GRI) (2013): G4 Sustainability Reporting Guidelines, Paper retrieved 10 February, 2014 from https://www.globalreporting.org/resourcelibrary/ GRIG4-Part1-Reporting-Principles-and-Standard-Disclosures.pdf.

GOETSCH, D.L. \& DAVIS, S.B. (2014): Quality Management for Organizational Excellence, Pearson, London.

GONZALO, J.A. \& PÉREZ, J. (2017): "Una propuesta de normalización relativa al valor añadido como medida alternativa de rendimiento empresarial", XIX Congreso Internacional AECA, 2017. 
GRÖNROOS, C. \& VOIMA, P. (2013): “Critical service logic: making sense of value creation and co-creation", Journal of the Academy of Marketing Science, 41(2), 133-150, D0I: 10.1007/ s11747-012-0308-3.

GROTH, J.C., BYERS, S.S. \& BOGERT, J.C. (1996): “Capital, economic returns and the creation of value", Management Decision, 24(6), 21-30, DOI: 10.1108/00251749610121452

GUASCH, S. (2015): “Contabilidad Simultánea: Valoración y Control de los Intangibles en la Gestión Integral, ACCID, Barcelona.

GUI, B. (1991): "The economic rationale for the Third Sector", Annals of Public and Cooperative Economics, 61, 4, 551-572, D0I: 10.1111/j.1467-8292.1991.tb01367.x.

HARRISON, J.S., BOSSE, D.A. \& PHILLIPS, R.A. (2010): "Managing for stakeholders, stakeholder utility functions, and competitive advantage", Strategic Management Journal, 31(1), 58-74, DOI: $10.2307 / 27735509$.

HARRISON, J.S. \& WICKS, A.C. (2013): "STAKEHOLDER THEORY, VALUE, AND FIRM PERFORMANCE", BUSIness Ethics Quarterly, 97-124, D0I:10.5840/beq20132314.

HAUSMAN, D. \& MCPHERSON, M. (2006): “Utilitarianism and consequentialism”, In: Economic Analysis, Moral Philosophy and Public Policy, 99-116, Cambridge University Press, Cambridge, DOI:10.1017/CB09780511754289.010.

JENSEN, M.C. (2001): "Value maximization, stakeholder theory, and the corporate objective function”, European Financial Management, 7, 297-317, D0I: 10.1111/1468-036X.00158.

KAPLAN, R. \& NORTON, D. (1992): "El cuadro de mando integral: medidas que impulsan el rendimiento", Harvard Business Review.

LAPLUME, A.O., SONPAR, K. \& LITZ, R.A. (2008): "Stakeholder theory: reviewing a theory that moves us", Journal of Management, 34(6), 1152-1189, D0I : 10.1177/0149206308324322.

LAZCANO, L., SAN-JOSE, L. \& RETOLAZA, J.L. (2019): "Social accounting in the social economy: a case study of monetizing social value". In: Modernization and Accountability in the Social Economy Sector, 132-150, IGI-Global, DOI: 10.4018/978-1-5225-8482-7.

LEWIS, B.R. \& GABRIELSEN, G.O. (1998): "Intra-organisational aspects of service quality management: the employees' perspective", The Service Industries Journal, 18 (2), 64-89, DOI: $10.1080 / 02642069800000019$.

MAHAJAN, G. (2010): Total Customer Value Management : Transforming Business Thinking, SAGE Publication. 
ORLITZKY, M., SCHMIDT, F.L. \& RYNES, S.L. (2003): “Corporate Social and Financial Performance: A Meta-analysis", Organization Studies, 24(3), 403-441, DOI: 10.1177/0170840603024003910.

PARASURAMAN, A., ZEITHAML, V.A. \& BERRY, L.L. (1988): “Servqual: a multiple-item scale for measuring consumer perception", Journal of Retailing, 64(1), 12.

PARASURAMAN, A., BERRY, L.L. \& ZEITHAML, V.A. (1991): "Refinement and reassessment of the SERVQUAL scale", Journal of Retailing, 67(4), 420-450.

RETOLAZA, J.L. \& RUIZ-ROQUEÑI, M. (2018): "El valor emocional", Ponencia presentada en los cursos de verano de la UPV/EHU, junio 2018.

RETOLAZA, J.L., SAN JOSÉ, L., RUIZ-ROQUEÑI, M., BARRUTIA, J. \& BERNAL, R. (2013): Metodología para la cuantificación del valor social generado por una organización. El caso de Lantegi Batuak. Disponible en: https://cutt.ly/BgClx6K.

RETOLAZA, J.L., SAN JOSÉ, L., RUIZ-ROQUEÑI, M. \& BARRUTIA, J. (2014): “Cuantificación del valor social: propuesta metodológica y aplicación al caso de Lantegi Batuak", Zerbitzuan: Gizarte Zerbitzuetarako Aldizkaria - Revista de Servicios Sociales, 55, 17-33.

RETOLAZA, J.L., SAN-JOSE, L. \& RUIZ-ROQUEÑI, M. (2015): "Monetarizing the social value: theory and evidence", CIRIEC-España, Revista de Economía Pública, Social y Cooperativa, 83, 43-62.

RETOLAZA, J.L., SAN-JOSE, L. \& RUIZ-ROQUEÑI, M. (2016): Social Accounting for Sustainability: Monetizing the Social Value, Springer, Cham, D0I: 10.1007/978-3-319-13377-5.

RUMELT R.P., SCHENDEL, D.E. \& TEECE, D.J. (1994): "Fundamental Issues in Strategy: A Research Agenda", Harvard Business School Press, Boston, MA.

SANDEL, M. (2012) : What Money Can't Buy: The Moral Limits of Markets, Allen Lane, London.

SATZ, D. (2010): Why Some Things Should not Be for Sale: The Moral Limits of Markets, 0xford University Press, Inc., New York.

SCHUMPETER, J. (1909): "On the concept of social value", The Quarterly Journal of Economics, 23(2), 213-232, DOI : $10.2307 / 1882798$.

SMITH, J.B. \& COLGATE, M. (2007): “Customer value creation: a practical framework", Journal of Marketing Theory and Practice, 15 (1), 7-23, D0I : 10.2753/MTP1069-6679150101.

SWEENEY, J.C. \& SOUTAR, G.N. (2001): "Consumer perceived value: the development of a multiple item scale", Journal of Retailing, 77 (2), 203-220, D0I: 10.1016/S0022-4359(01)00041-0.

SULLIVAN, P.H. (2000): Value Driven Intellectual Capital: How to Convert Intangible Corporate Assets into Market Value, John Wiley \& Sons, Inc. 
ULRICH, P. \& FIBITZ, A. (2018): "Corporate governance mechanisms in family firms - A socioemotional wealth perspective", Corporate Ownership \& Control, 15(3), 32-46, D0I:10.22495/ cocv15i3art3.

VARGO, S.L. (2008): "Customer integration and value creation: paradigmatic traps and perspectives", Journal of Service Research, 11(2), 211-215, D0I: 10.1177/1094670508324260.

VARGO, S.L. \& LUSCH, R.F. (2008): "Service-dominant logic: continuing the evolution", Journal of the Academy of Marketing Science, 36(1), 1-10, DOI: 10.1007/s11747-007-0069-6.

VARGO, S.L. \& LUSCH, R.F. (2008): "From goods to service (s): divergences and convergences of logics", Industrial Marketing Management, 37(3), 254-259, DOI: 10.1016/j.indmarman.2007.07.004.

VENKATESAN, R. \& KUMAR, V. (2004): "A customer lifetime value framework for customer selection and resource allocation strategy", Journal of Marketing, 68(4), 106-125, D0I: 10.1509/ jmkg.68.4.106.42728.

WHITEHEAD, G. (1996): Economics, 15th edn: Butterworth-Heinemann, Oxford.

WILLIAMSON, 0. (1984): “Corporate governance”, Yale Law Journal, 93, 1197-1230, DOI: $10.2307 / 796256$.

WOODALL, T. (2003): “Conceptualising 'value for the customer': an attributional, structural and dispositional analysis", Academy of Marketing Science Review, 12(1), 1-42.

YIN, R.K. (2014): Case Study Research Design and Methods, Sage Publications, London.

ZEITHAML, V.A. (1988): “Consumer perceptions of price, quality, and value: a means-end model and synthesis of evidence", Journal of Marketing, 52(3), 2-22, D0I: 10.1177/0022242988 05200302 .

ZEGHAL, D. \& MAALOUL, A. (2010): "Analysing value added as an indicator of intellectual capital and its consequences on company performance", Journal of Intellectual Capital, DOI: $10.1108 / 14691931011013325$.

ZELLWEGER, T.M. \& ASTRACHAN, J.H. (2008): “On the emotional value of owning a firm”, Family Business Review, 21(4), 347-363, D0I: 10.1111/j.1741-6248.2008.00129.x.

ZERBE, R.O. \& BELLAS, A.S. (2006): A Primer for Benefit-Cost Analysis, Edward Elgar Publishing, Northampton.

ZERBE, R.0. (2004): "Should moral sentiments be incorporated into benefit-cost analysis? An example of long-term discounting", Policy Sciences, 37, 305-318, DOI: 10.1007/s11077-0055750-3. 